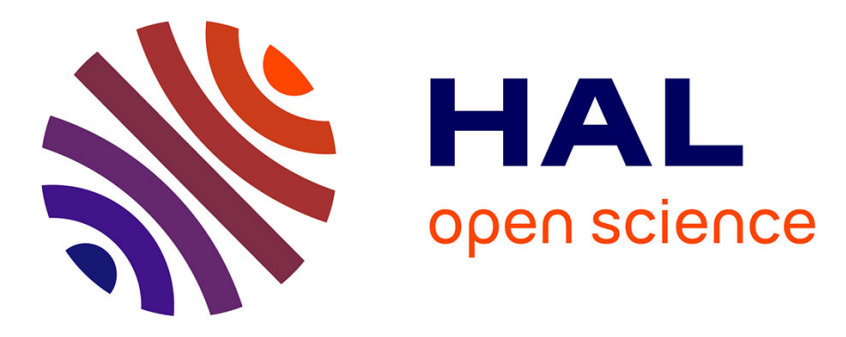

\title{
Simulation of microalgae growth in limiting light conditions: Flow effect
}

Jeremy Pruvost, J. Legrand, P. Legentilhomme, A. Muller-Feuga

\section{To cite this version:}

Jeremy Pruvost, J. Legrand, P. Legentilhomme, A. Muller-Feuga. Simulation of microalgae growth in limiting light conditions: Flow effect. AIChE Journal, 2002, 48 (5), pp.1109-1120. 10.1002/aic.690480520 . hal-02534009

\section{HAL Id: hal-02534009 \\ https://hal.science/hal-02534009}

Submitted on 17 Apr 2020

HAL is a multi-disciplinary open access archive for the deposit and dissemination of scientific research documents, whether they are published or not. The documents may come from teaching and research institutions in France or abroad, or from public or private research centers.
L'archive ouverte pluridisciplinaire HAL, est destinée au dépôt et à la diffusion de documents scientifiques de niveau recherche, publiés ou non, émanant des établissements d'enseignement et de recherche français ou étrangers, des laboratoires publics ou privés. 


\title{
Simulation of Microalgae Growth in Limiting Light Conditions: Flow Effect
}

\author{
J. Pruvost, J. Legrand, and P. Legentilhomme \\ GEPEA Laboratory, University of Nantes, CRTT-IUT, F-44602 Saint-Nazaire Cedex, France
}

A. Muller-Feuga

PBA Laboratory, IFREMER, 44311 Nantes Cedex 3, France

\begin{abstract}
Effect of hydrodynamical conditions on a microalgae culture growth was investigated in a photobioreactor with annular light chambers, with the focus on the relation between the cell displacement and the amount of light received by microorganisms, by comparing two different flow conditions in light chambers: an axial flow generating a poor radial mixing and a 3-D swirling motion. To determine microorganism trajectories, a Lagrangian approach was retained, allowing light received to be considered from a single microalga point of view. The light distribution was calculated using Beer-Lambert law, and a biological modeling of the culture growth was proposed, with consideration of light/dark cycle effects induced by cell displacement in the depth of the culture. Finally, batch cultures of Porphyridium purpureum were simulated for both hydrodynamical conditions in light chambers. The advantage of applying a three-dimensional motion to generate cell renewal in front of the light source, allowing microorganisms to use light more efficiently, is clearly shown.
\end{abstract}

\section{Introduction}

Because of their taxonomic diversity, microalgae are found to synthesize various high-value organic compounds that cannot be produced by the usual microorganisms commonly employed in bioreactors. Microalgae thus appear as a new natural resource with potential industrial interest. But their production under controlled conditions implies a dedicated reactor design called photobioreactors, which mainly differ from classic bioreactors, that is, fermentors or enzymatic reactors, by the need for a light supply in addition to classic chemical growth substrates, because microalgae are photoautotrophic microorganisms (Richmond, 1986; Pulz and Scheibenbogen, 1998; Muller-Feuga et al., 1998a; Grima et al., 1999). But light cannot be considered as a classic substrate, because it involves two antagonist phenomena: a high amount of photonic energy is requested to ensure microorganisms growth conditions, but the increase of the concentration in the process decreases the depth of light penetration in the culture, due to the cell self-shading. Light use by the culture is thus difficult to optimize and the microalgae concentration in the process remains limited, making it of too low economical in- terest for industrial applications (Grima et al., 1999; Janssen et al., 1999; Fernandez et al., 1997; Vonshak, 1997; Chen and Lee, 1997; Richmond, 1996). A research effort in engineering is needed to design optimized processes able to produce concentrated microalgae cultures of high quality and of sufficiently high productivity.

A way to improve photobioreactor efficiency is to decrease the light limitation by increasing the intensity of the light supply. But microalgae, which grow naturally under the sea surface, are sensitive to high light intensity due to the socalled photolimitation phenomenon (Richmond, 1986; Grima et al., 1996). Because of the strong light gradient that appears in the culture, the amount of phototonic energy efficiently used is often very low: microalgae located near the light source are too highly illuminated, while those deeper in the culture and far from the light source do not receive enough light energy to ensure good photosynthetic conditions (Grima et al., 1996). Only an intermediate part of the culture is ideally illuminated. The solution of increasing the light supply is thus of limited value.

A more promising solution investigated in this study is to improve light availability by optimizing hydrodynamical con- 
ditions in the light chamber of the reactor. A three-dimensional (3-D) motion can be employed to promote a microalgae displacement along the light gradient in the culture, allowing cell renewal in front of the light supply. There are some examples of photobioreactors where this kind of flow is involved, as in the work of Carlozzi and Torzillo (1996), who used a curved tubular geometry to generate secondary flow motion in bends (Dean-type vortices), while Miller et al. (1964) designed an annular photobioreactor with a rotating cylinder to induce Taylor-type vortices. However, only few studies have been done in order to accurately characterize the influence of hydrodynamics on microalgae light availability and photobioreactors efficiency, even though the beneficial effects of this parameter are already established. By studying the effects of flow rate, Richmond (1996) deduced the importance of generating a turbulent motion to improve the light access of flowing microalgae, and thus the productivity of the photobioreactor. Other studies dealing with the influence of a turbulent motion are available (Chen and Lee, 1997; Carlozzi and Torzillo, 1996; Grima et al., 1999; Janssen et al., 1999; Grobbelaar, 1994; Thomas and Gibson, 1990), but it seems that the effects of a turbulent flow remain difficult to characterize with accuracy in real culture conditions. An increase in the flow rate does not necessarily lead to an improved process efficiency, because some undesirable physiological responses of the microorganisms can arise. For example, microalgae are found to be especially sensitive to hydrodynamic stress (Vandanjon et al., 1999; Jaouen et al., 1999; Baldyga and Pohorecki, 1998; Mitsuhashi et al., 1995; Carlozzi and Torzillo, 1996; Gudin and Chaumont, 1991); also, an increase in mixing can lead to a negative effect on the culture growth. Thus, it is difficult to experimentally investigate the influence of a unique parameter such as hydrodynamics because of the complex biological behavior of $\mathrm{mi}$ croalgae: a change in flow motion in the process does not only affect the light availability but can induce other uncontrolled phenomena, like microorganism sensitivity to hydrodynamical stress or mass-transfer modifications between cells and the surrounding medium (Grobbelaar, 1994; Merchuk et al., 1998).

The aim of this study is to focus on the interest of changing flow conditions in the process in order to improve the light availability, and thus its use by microalgae. Because mixing cannot be experimentally improved without generating additional undesirable effects, the establishment of a photobioreactor model is the way to decouple the influence of the different parameters and to restrict our attention to the effect of three-dimensional flows on the improvement of light availability for microalgae cultures. This approach should provide a first assessment of the theoretical benefit of applying a 3-D motion in such a photobioreactor, before attempting further optimization of the flow conditions to improve the light use in photobioreactors.

In the photobioreactor under study, light is provided in tubular geometries called light chambers that use fluorescent tubes to illuminate the surrounding culture (Figure 1), and the 3-D motion used here is an annular swirling decaying flow induced by a tangential inlet. Although other methods are available for generating 3-D motion in such geometries (guide vanes or direct rotation of the elements), a tangential inlet can be adapted to annular geometries using a rather simple

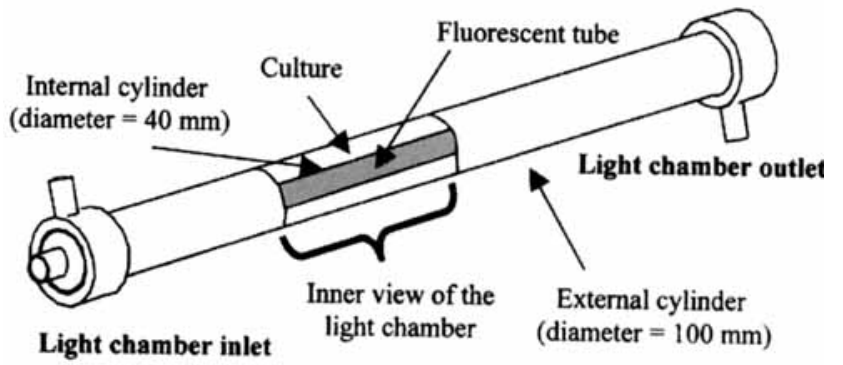

Figure 1. Annular light chamber.

design (Gupta et al., 1984; Legentilhomme and Legrand, 1990, 1991; Legentilhomme et al., 1993). Only the inlets of the photobioreactor have to be modified to turn the axial flow into a 3-D swirling one. The tangential inlet and a sketch of the induced swirling flow are given in Figure 2.

The model developed in this work refers to some previous investigations that were specifically aimed at achieving the simulation of culture growth. Because these studies are described elsewhere, only general procedures will be summarized in the following sections, but complete descriptions can be found in corresponding articles (Pruvost et al., 2000, 2001, 2002). The present work focuses on the simulation procedure and especially on new aspects of the simulation, namely the determination of the light gradient as a function of the culture concentration and the biological modeling of microalga Porphyridium purpureum photosynthetic response. Finally, to characterize the influence of hydrodynamical conditions on

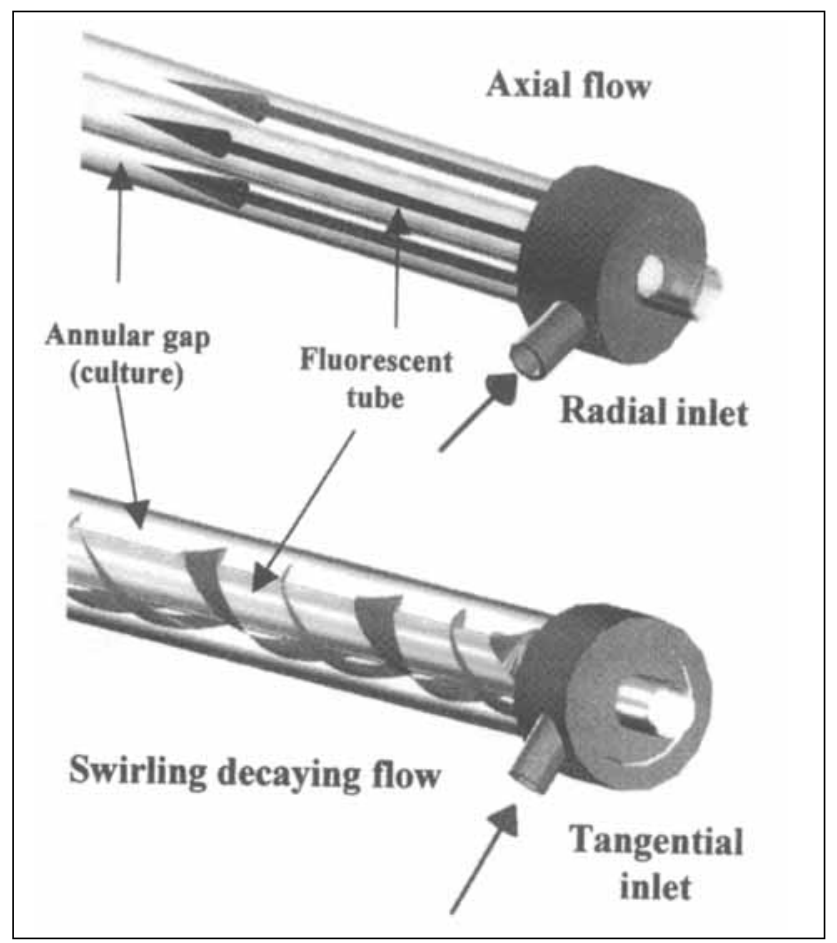

Figure 2. Axial and swirling flows. 
microalgae growth, batch cultures of $P$. purpureum will be simulated in a light chamber using a swirling decaying flow. The same study is also performed in a photobioreactor light chamber characterized by an axial flow induced by a radial inlet, which is commonly used in photobioreactors of annular geometries (Figure 2). Such a flow involving the poor displacement of the microalgae along the light gradient, where the results in both hydrodynamical conditions are compared, will show the benefit of a 3-D displacement of microalgae in order to improve the light use.

\section{Modeling of the Growth of Porphyridium purpureum}

\section{Photobioreactor description}

This study is applied to a photobioreactor patented by the French Research Institute for Exploitation of the Sea (IFREMER) (Muller-Feuga et al., 2000). The process was designed to make microalgae culture possible under fully controlled and axenic conditions, which are necessary for the production of high-value molecules. Further details on this process can be found in Muller-Feuga et al., 1998a,b). The photobioreactor is presented in Figure 3. Artificial light is provided in a series of eight annular geometries, so-called light chambers, each of which contains a central fluorescent tube that illuminates the surrounding culture flowing the annular gap. A circulation loop is used in the photobioreactor, where the culture crosses each of the eight light chambers at the rear of the photobioreactor in succession and then moves to the dark front side, where nutrients and substrates are provided. The light chambers are $1.5 \mathrm{~m}$ long, and the diameters of internal and external cylinders are $40 \mathrm{~mm}$ and 100 $\mathrm{mm}$, respectively, which corresponds to an annular gap width of $30 \mathrm{~mm}$.

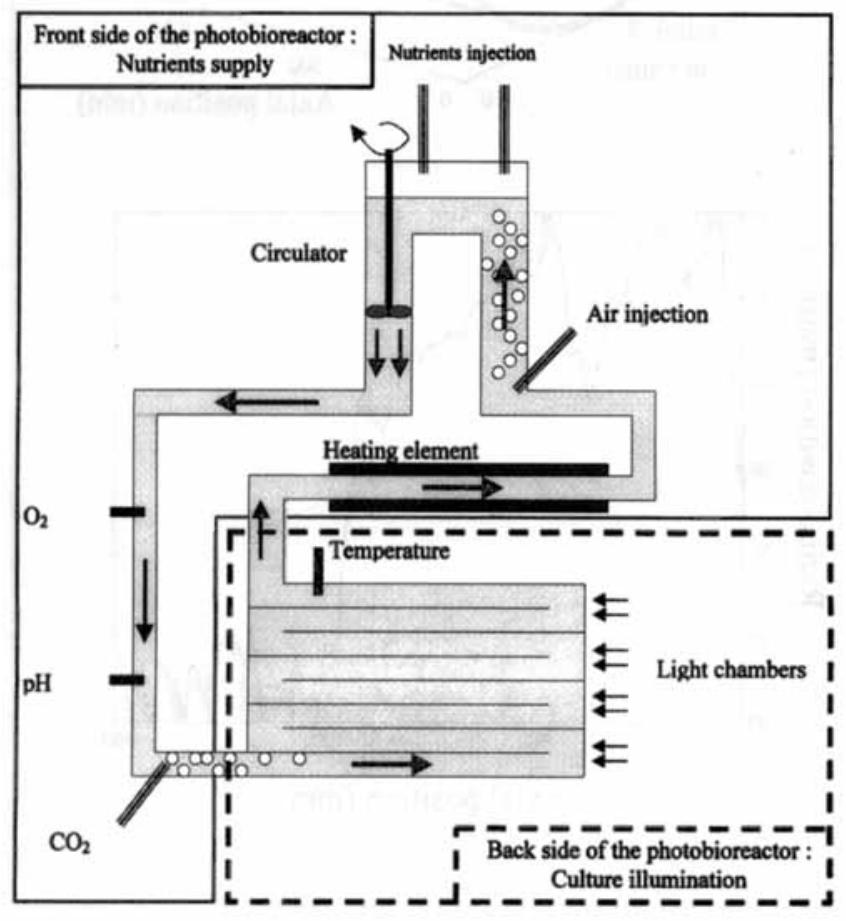

Figure 3. Photobioreactor.
Previous investigations of hydrodynamical characteristics were carried out in an experimental setup that reproduces one of the photobioreactor light chambers. Measurements of the hydrodynamical characteristics were made using the particle image velocimentry (PIV) technique for the flow rate, $Q=1,190 \mathrm{~L} \cdot \mathrm{h}^{-1}$. Both the axial and swirling flows were induced by alternating inlet conditions, which are, respectively, radial, with a diameter of $65 \mathrm{~mm}$, and tangential, with a diameter of $45 \mathrm{~mm}$. The measurement of hydrodynamical values using PIV is described in Pruvost et al. (2000).

\section{Photobioreactor modeling: the Lagrangian approach}

Attempts at modeling photobioreactors mainly investigate the influence of parameters such as $\mathrm{pH}$ or nutrient concentration, which can be assumed to be homogeneously distribution (Frohlich et al., 1983; Yokota et al., 1994). But the same assumption cannot be applied to light because of the light attenuation. If the light influence is approximated, the model efficiency remains limited, as shown by Grima et al. (1996), who pointed out that difficulties arise for photobioreactors scaleup. This is mainly due to the biological photosynthetic response of microalgae, which is very sensitive to the conditions of light reception. The culture in the light chamber has a depth for which the light received by the microalgae is optimal, with photoinhibition for positions nearer the light source, and too little illumination deep inside the culture (Merchuk et al., 1998). An accurate representation of microorganism growth is not obtained when a mean illumination intensity is considered because of the difference in physiological behavior in the same culture due to the light gradient. Thus, both microalgae motion and light distribution in a model must be considered.

The experimental investigation using PIV has shown the complex behavior of the swirling flow field due to the threedimensional motion involved and the free decay of the swirl intensity along the axial coordinate, making hydrodynamical conditions not fully developed and asymmetric (Pruvost et al., 2000). A Lagrangian approach allows the calculation of accurate microorganism trajectories in the light chambers, starting from the entrance of each light chamber and then determining the successive positions followed by a microalga. The light received by a microalga can be deduced, giving an accurate relation between hydrodynamics and the received energy. A method was developed for calculating the trajectories (Pruvost et al., 2002), using the results of the PIV flow-field measurements (Pruvost et al., 2000).

For microorganisms having the same density as the fluid, effects of mass or buoyancy forces on trajectories were neglected. Correction of pathlines that arises when particles are of greater size than the smallest flow eddies also were not considered (Ormancey and Martinon, 1984), cells size being ca. some tens of micrometers, with the smallest eddy size given by the Kolmogorov scale ca. a hundred micrometers in duct flows (Bradshaw, 1971). It was thus assumed that microalgae follow the same trajectories as elementary fluid particles, and the method described in Pruvost et al. (2002) was applied without correction.

Because of the spatial dependency of the swirl motion, the Lagrangian approach requires knowledge of the flow-field characteristics on the entire geometry, which were recon- 
structed from PIV data with an interpolation method based on a neural-network technique with radial basis function. Unlike the classic numerical interpolating scheme, the neuralnetwork technique considers the nonlinear evolution of data (Powell, 1987) as it occurs in a 3-D flow field. An efficient reconstruction of the evolution of hydrodynamical quantities can be obtained using only a small number of the original values. Pruvost et al. (2001) have used this method with PIV measurements to determine the swirling decaying flow characteristics in the entire geometry. A mathematical representation of the mean velocity and turbulent intensity for the three components of the velocity field was finally obtained, allowing the fluid trajectories to be calculated. Two examples of trajectories are given in Figure 4, one for the swirling motion and the second for the axial one. Significant differences between the two trajectories appear, regarding the displace- ment along the radial coordinate (i.e., along the light gradient) in particular. A cell renewal can be expected in swirling flows, whereas the axial flow involves the main displacement parallel to the light-chamber axis.

\section{Modeling of the light received by microalgae}

If light is considered the only limiting parameter, microalgae growth depends on the amount of photonic energy received for the photosynthesis process. To determine this energy, the light distribution in the culture has to be calculated first. Many studies are available in the literature for photochemical reactors, and specific references can be found in Cassano et al. (1995) or in our previous study (Pruvost et al., 1999). Common methods developed for photoreactors are applicable in the photobioreactor case (Yokota et al., 1994;

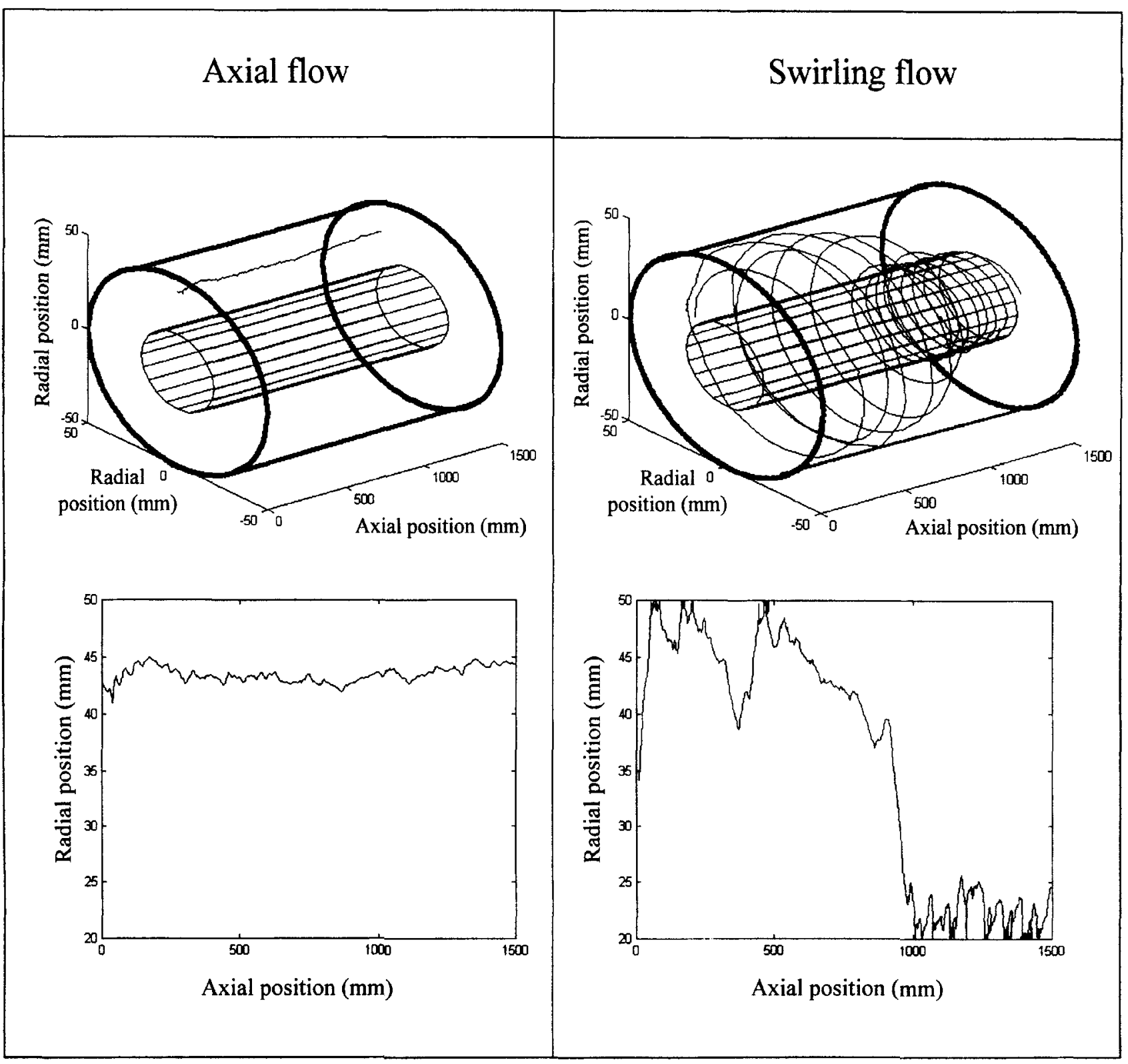

Figure 4. Examples of trajectories obtained using the Lagrangain calculation method. 
Grima et al., 1996; Fernandez et al., 1997). The complexity of determining the light distribution is directly linked to the geometry of the reactor and the position of light source supplies. In the case of the photobioreactor under study, this problem remains simple because of the cylindrical symmetry involved in the use of fluorescent tubes axially centered in the annular light chambers. For the given culture concentration and position in the geometry, the light distribution in the culture is a function of both the light-emitting source and the absorbing medium. For photobioreactor applications, the light attenuation in the culture is commonly represented by the so-called Beer-Lambert law (Muller-Feuga et al., 1998b; Grima et al., 1996, 1999; Frohlich et al., 1983; Pulz and Scheibenbogen, 1998; Cornet et al., 1995; Lee, 1999). However, this approach can remain too simple for microalgae culture because light absorption is considered without scattering effects. The influence of scattering effects depends on the optical properties and sizes of the cells and of the microalga species cultivated. If those scattering effects cannot be neglected, other models are available (Cornet et al., 1998; Cassano et al., 1995), but the benefit of using the Beer-Lambert law, which represents light attenuation with a rather simple equation, is lost. Indeed, a complete formulation with accurate representation of the scattering effects can involve a numerical resolution, with finite difference method, for instance, making light distribution time-consuming and difficult to obtain (Cornet et al., 1998; Cassano et al., 1995). Intermediate solutions can be found in the literature. For example, by assuming that scattering occurs in microalgae culture only along the axis of light propagation, Cornet et al. (1995) proposed a simplified solution of the radiative transfer equation. But for such an approach, the scattering properties of microalgae have to be known, and these are difficult to measure experimentally. For this reason, and because the aim of this study is to investigate the influence of hydrodynamical conditions on cell light accessibility and on culture growth, the Beer-Lambert law was used only in the first assumption. The simple representation of light attenuation allows rapid calculation of the light received by cells, which is important for the proposed Lagrangian simulation of culture growth that induces the photonic energy distribution to be redetermined for each new evolution of the culture concentration. Finally, as shown later, the exponential decrease of light intensity with the increase in culture concentration obtained by using the Beer-Lambert law will be experimentally observed in real culture conditions, allowing an accurate representation of the light distribution.

Because of the cylindrical geometry, the light attenuation in the Beer-Lambert law can be assumed to only occur in the radial direction. For polychromatic light sources like fluorescent tubes, the light spectrum is divided into wavelength intervals, and the Beer-Lambert law is then expressed by

$$
\frac{I(r, C, \Delta \lambda)}{I_{0}(C, \Delta \lambda)}=10^{-\epsilon_{\lambda \lambda} C\left(r-R_{i}\right)}
$$

where $r$ is the radial position in the geometry, $R_{i}$ is the radius of the internal cylinder of the annular light chamber, $C$ is the culture concentration expressed in dry weight, $I(r, \Delta \lambda)$ and $I_{0}(\Delta \lambda)$ are, respectively, the light intensity received at a position $r$ and emitted by the light source for a given wavelength interval $\Delta \lambda$, and $\epsilon_{\Delta \lambda}$ is the absorption coefficient for the wavelength interval $\Delta \lambda$.

Two types of fluorescent-tube light sources are used in the studied photobioreactor: daylight tubes and Grolux tubes from Sylvania. They are both the same length $(1.5 \mathrm{~m})$, but their light spectra differ; these were chosen for their complementary stimulation of microalgae photosynthesis pigments. The light spectra of both sources are given in Figure 5: the light-emitting power of Grolux- and daylight Satin-type tubes being, respectively, $175 \mu$ Einstein $\cdot \mathrm{m}^{-2} \cdot \mathrm{s}^{-1}$ and $236 \mu$ Einstein $\cdot \mathrm{m}^{-2} \cdot \mathrm{s}^{-1}$.

Absorption coefficients $\epsilon_{\Delta \lambda}$ are a function of the absorbing medium. Values of $\epsilon_{\Delta \lambda}$ were determined for the P. pur-

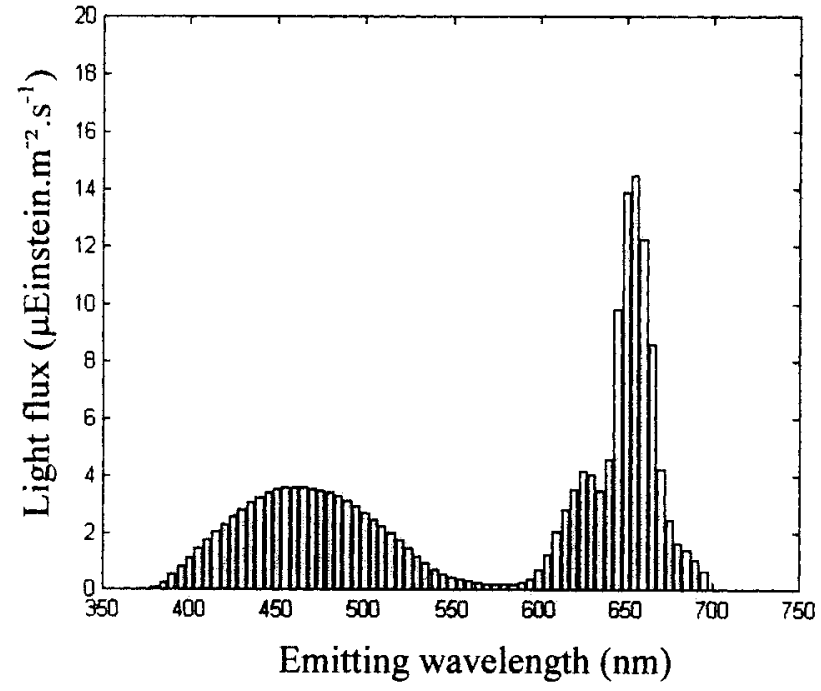

(a)

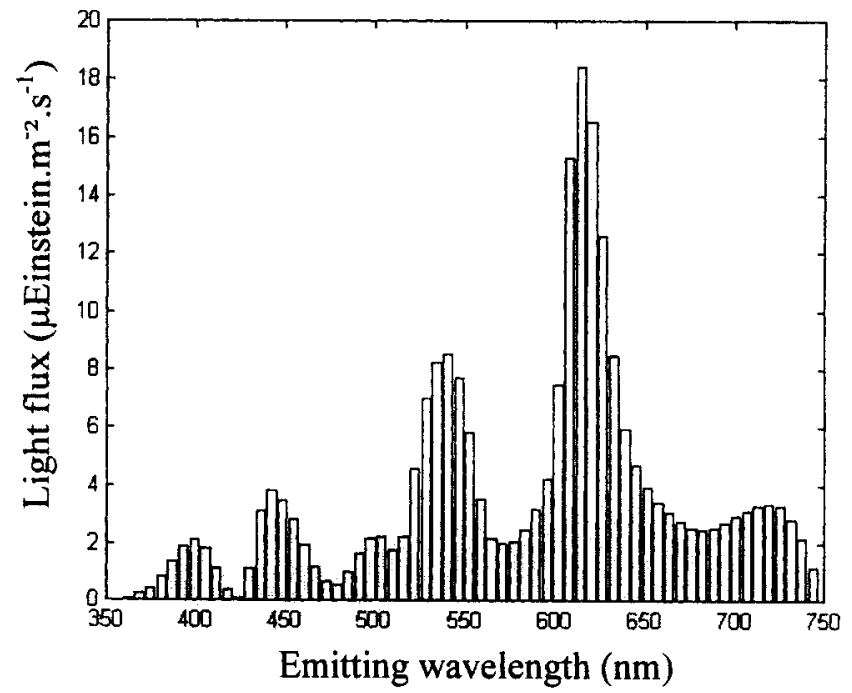

(b)

Figure 5. Emitting spectra of both light sources discretized on emitting wavelength intervals: (a) Grolux-type tube; (b) satin-type tube. 


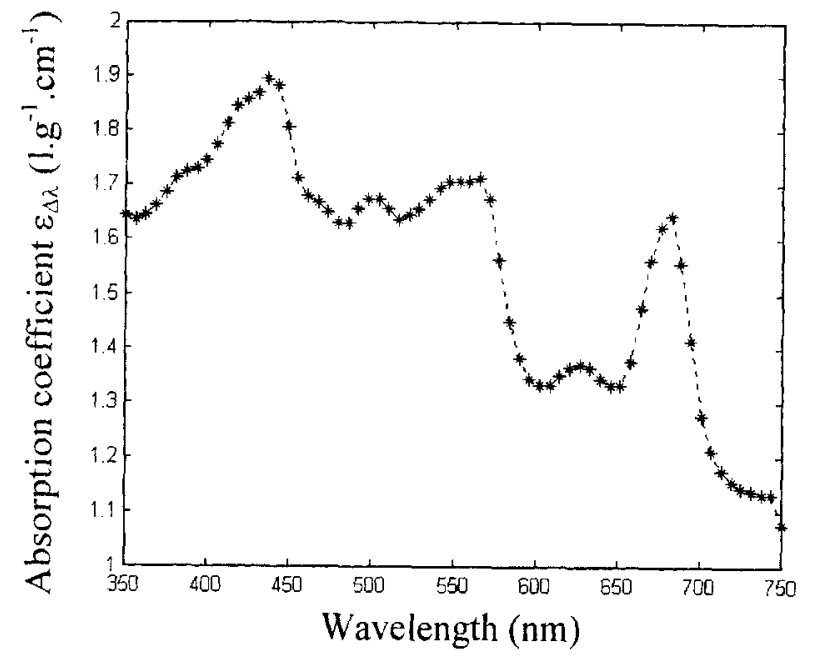

Figure 6. Absorption coefficients obtained on emitting wavelength intervals.

pureum culture by using a spectrophometer to measure the absorption spectra of some microalgae aliquots of different dry-weight concentrations. These values were next deduced from the following equation:

$$
D O_{\Delta \lambda}=\epsilon_{\Delta \lambda} C l_{c}
$$

where $D O_{\Delta \lambda}$ is the optical density measured for the wavelength interval $\Delta \lambda$, and $l_{c}$ is the length of the spectrophotometer measurement cell $\left(l_{c}=1 \mathrm{~cm}\right)$.

By measuring the experimental absorption spectra for different dry-weight culture concentrations, the values of $\epsilon_{\Delta \lambda}$ plotted in Figure 6 were obtained by linear fitting with an error of less than $10 \%$ on the experimental results. The depth of light penetration in the culture can be deduced for each wavelength interval, $\Delta \lambda$, for a given dry-weight concentration $C$. Figure 7 shows an example of the light-emitting spectrum evolution obtained for $C=0.5 \mathrm{~g} \cdot \mathrm{L}^{-1}$. The global value of the light intensity $I(r)$ that is received by a microalga located

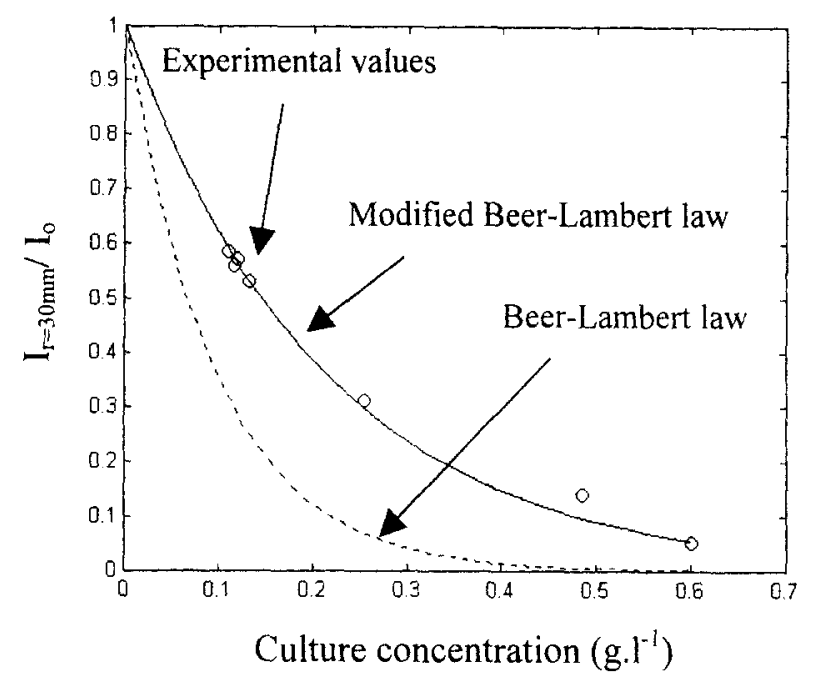

Figure 8. Beer-Lambert law results vs. experimental measurements.

at a given position $r$ is calculated as follows:

$$
I(r, C)=\sum_{\Delta \lambda} I_{0}(\Delta \lambda) \cdot 10^{-\epsilon_{\Delta \lambda} C\left(r-R_{i}\right)}
$$

The light-attenuation model is experimentally verified by determining the amount of light not absorbed by the culture. A quantum LICOR meter, located on the light chamber's outer cylinder and measuring in the photosynthetically active radiation (PAR) domain, was used to determine the transmitted amount of light for some culture dry-weight concentrations, and theoretical values of the light intensity were achieved by simply setting $r=R_{o}$ in Eq. 3, where $R_{o}$ is the radius of the external cylinder of the annular geometry. Calculated and experimental data are compared in Figure 8. It appears that the Beer-Lambert law overestimates the culture's real light absorption. This was already observed by Fernandez et al. (1997) for modeling light attenuation by the

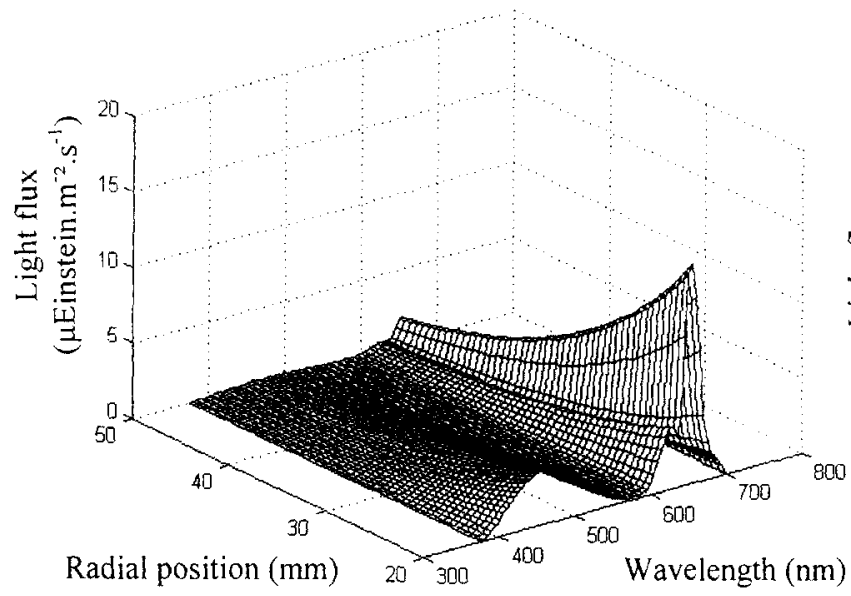

(a)

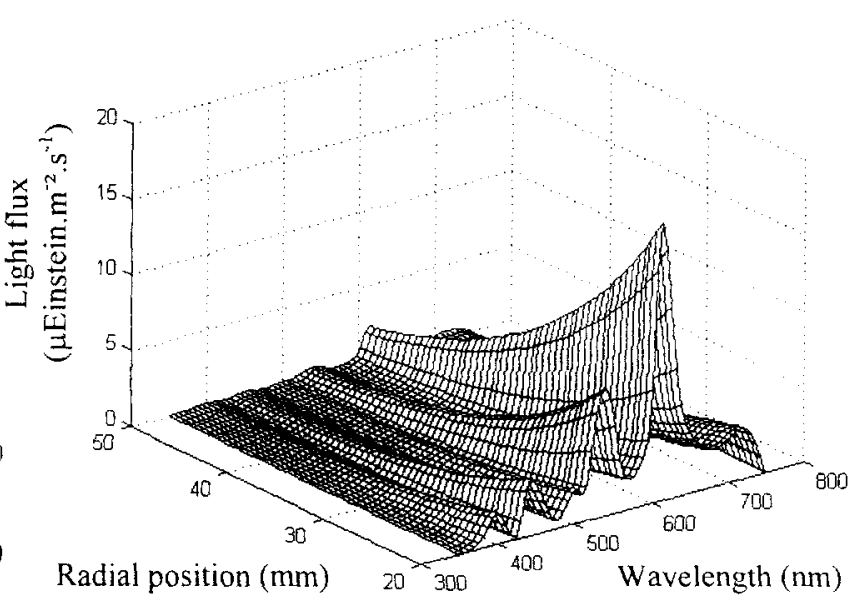

(b)

Figure 7. Emitting spectra attention in the culture for $C=0.5 \mathrm{~g} \cdot \mathrm{L}^{-1}$ : (a) Grolux-type tube; (b) satin-type tube. 


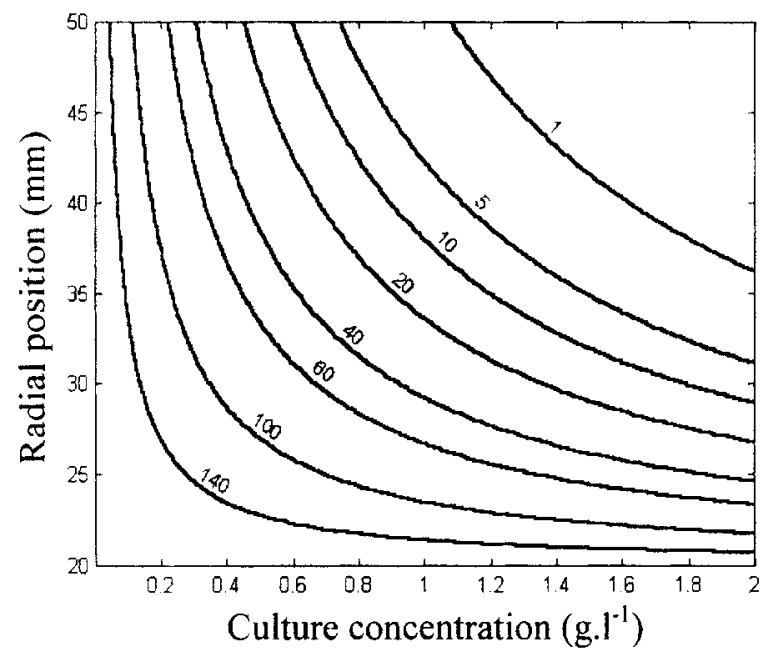

Figure 9. Isovalues of light flux (in $\mu$ Einstein $\cdot \mathrm{m}^{-2} \cdot \mathrm{s}^{-1}$ ) obtained for the Grolux-type tube in the culture as a function of culture dry-weight concentration and radial position.

Beer-Lambert law in the case of microalgae cultures. Deviation from the Beer-Lambert law is explained by the assumptions used for its establishment, such as negligible scattering effects due to reflecting particles, and a constant direction of the light beams while crossing the absorbing medium. As explained before, these conditions cannot be fulfilled in microalgae culture because of the scattering effects and an important light-diffusion, which depends on the optical properties of the culture (Fernandez et al., 1997; Cassano et al., 1995; Cornet et al., 1998). Nevertheless, the Beer-Lambert law's principle that there is an exponential decrease in light intensity remains applicable: only absorption coefficients $\epsilon_{\Delta \lambda}$ are overestimated. If Eq. 3 is modified to accurately represent the real light absorption, a good approximate representation of the light attenuation can be obained as follows:

$$
I(r, C)=\sum_{\Delta \lambda} I_{0}(\Delta \lambda) \cdot 10^{-t_{\mathrm{corr}} \epsilon_{\Delta \lambda} C\left(r-R_{i}\right)}
$$

where $t_{\text {corr }}$ is a correction term that is determined from the experimental values found in Figure 8.

Results calculated using $t_{\text {corr }}=0.45$ are presented in Figure 8 . Good agreement between the experimental data and the modified Beer-Lambert law is observed. Thus Eq. 4 will be used to determine the light intensity in the $P$. purpureum culture as a function of radial position and the dry-weight concentration of the culture. Results are presented in Figure 9 for the Grolux-type fluorescent tubes.

\section{Biological modeling of the growth of Porphyridium purpureum}

The growth of the culture can be obtained by using a biological growth model that gives the growth rate, $\mu$, as a function of the light intensity received, $I$. Some authors (Grima et al., 1996, 1999; Muller-Feuga, 1998; Pulz and Scheinbenbogen, 1998) have proposed different expressions of the $\mu(I)$ function. A representative description and comparison can be found in Muller-Feuga (1998), who proposed a model that

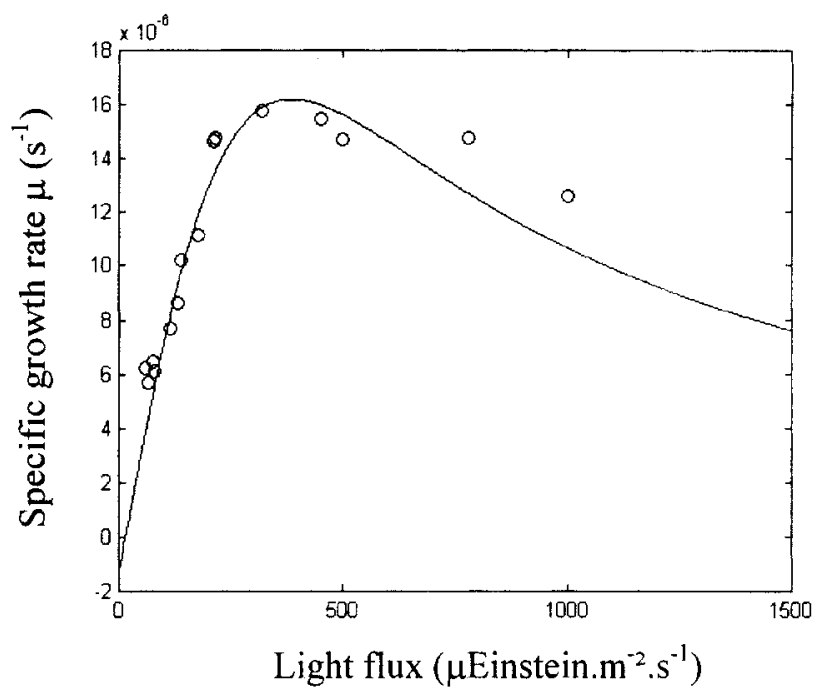

Figure 10. Culture-specific growth rate $\mu(I)$ using the Muller-Feuga (1998) equation fitted with data from Dermoun et al. (1992) for $P$. purpureum at $25^{\circ} \mathrm{C}$.

was established for $P$. purpureum. The model expresses the main aspects of the photosynthetic response of the microalgae to light, namely, the photoinhibition of the cells to high values of $I$, and the weight loss that occurs below a certain $I$ value called the compensation light energy, $I_{e}$. The mathematical expression of this model is

$$
\mu=\frac{1 d C}{C d t}=\frac{2 \mu_{s}\left(1-\rho_{e}\right)\left(\rho-\rho_{e}\right)}{\left(1-\rho_{e}\right)^{2}+\left(\rho-\rho_{e}\right)^{2}}
$$

where $t$ is time, $\rho=I / I_{s}$ is the normalized light intensity, $\rho_{e}$ $=I_{e} / I_{s}$ is the normalized light intensity compensation, $I_{s}$ is the light intensity received at saturation, and $\mu_{s}$ is the specific growth rate at saturation. The three required parameters, $I_{s}, I_{e}$, and $\mu_{s}$, were deduced from the experimental results of Dermoun et al. (1992), obtained for $P$. purpureum at $25^{\circ} \mathrm{C}$. The used light intensity range was substituted into Eq. 5 (i.e., $I$ less than $236 \mu$ Einstein $\cdot \mathrm{m}^{-2} \cdot \mathrm{s}^{-1}$ ), giving $I_{e}=15$ $\mu$ Einstein $\cdot \mathrm{m}^{-2} \cdot \mathrm{s}^{-1}, I_{s}=385 \mu$ Einstein $\cdot \mathrm{m}^{-2} \cdot \mathrm{s}^{-1}$, and $\mu_{\mathrm{s}}=$ $16 \times 10^{-6} \cdot \mathrm{s}^{-1}$. The corresponding results of the growth rate are presented in Figure 10.

By using Eq. 4 to determine the light intensity $I(r, C)$, the specific growth rate $\mu(I)$ can be deduced as a function of the culture's dry-weight concentration, and Eq. 5 can be used to deduce the radial position. Results are shown in Figure 11 for the Grolux-type, and it can be seen that for high culture concentrations, regions with negative growth rate are located deep in the culture. However, using Eqs. 4 and 5 to determine the growth rate of the culture, $\mu(I)$, is not sufficient to represent the real microalgae photosynthetic growth, because the biological response as represented by Eq. 5 can be applied only to a low culture concentration, for which there is no self-shading of cells to allow each microalga to receive constant illumination. It is well known that microalgae in photobioreactors are subject to light intensity variations due to the light gradient related to cell self-shading and to mi- 


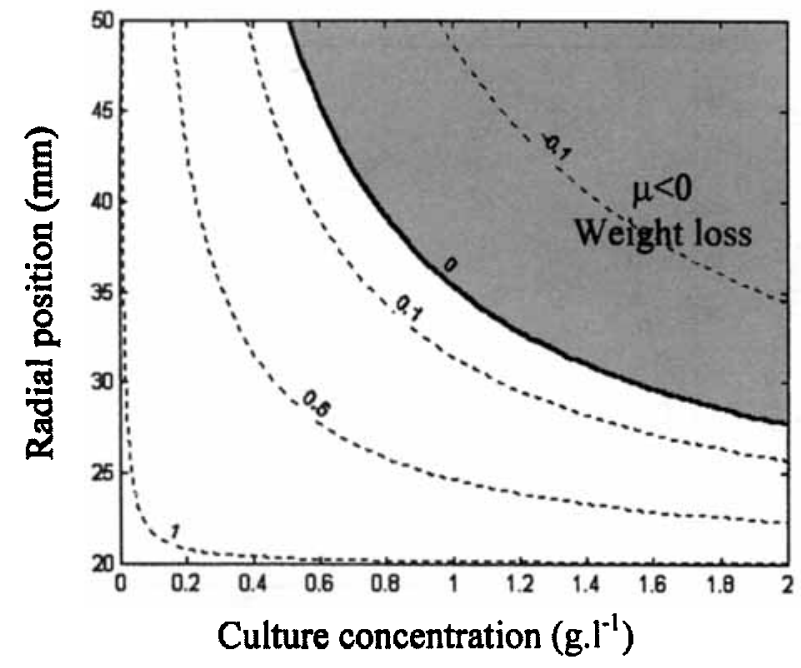

Figure 11. Isovalues of the culture specific growth rate (in $\mathbf{s}^{-1}$ ) for the Grolux-type tube as a function of culture dry-weight concentration and radial position.

croalgae displacement. The model was modified to take these effects of light variations on microalgae growth into account.

Photosynthesis appears as a complex dynamic mechanism that results in various physiological responses to adapt and protect microalgae, for instance, from light variations (Vonshak, 1997). In particular, this is the so-called light/dark cycle effects, as observed in many studies (Grobbelaar, 1994; Grobbclaar et al., 1996; Lee and Lee, 2001; Pulz and Scheibenbogen, 1998; Merchuk et al., 1998; Janssen et al., 1999). Merchuk et al. (1998) have emphasized a particular biological behavior by $P$, purpureum. By investigating the influence of dark and light periods on culture growth, these authors found that this species has the capacity to remain for a while in dark zones without loss of productivity. They explained that phenomenon by a particular mechanism of the photosynthetic apparatus, which appears to be regulated by a protein named D1 (Aro et al., 1993). If a cell stays in a too highly illuminated region, D1 proteins are destroyed, which breaks the photosynthetic mechanism chain and leads to cessation of cell growth. Fortunately, these proteins are continuously synthesized by the microorganism, and after good illumination conditions are renewed, the photosynthetic system repairs itself. By allowing cells to flow in dark zones for a while, full destruction of the D1 protein is avoided and the photosynthetic apparatus remains effective (Merchuk et al,, 1998; Janssen et al., 1999). The presence of dark zones in photobioreactors is not necessarily negative, but corresponds to the culture's biological need for its photosynthetic apparatus to recover (Muller-Feuga et al., 1998b; Grima et al., 1996).

Following these findings, the Muller-Feuga model was adapted to take into account high concentration conditions according to the values obtained by Merchuk et al. (1998) for $P$. purpureum, who stated that a dark period of $6 \mathrm{~s}$ following a light period longer than $27 \mathrm{~s}$ did not influence the photobioreactor productivity. Thus, it was assumed in the final biological modeling that no negative growth rate $\mu$ was achieved in the dark area of the culture if the period during which the cell remains in that region was shorter than $6 \mathrm{~s}$, and the previous illumination period was greater than $27 \mathrm{~s}$. The effect of light/dark cycles on growth rate is shown in Figure 12.

\section{Simulation procedure}

The culture growth simulation algorithm is based on the Lagrangian trajectory calculation method. For batch-type cultures, Eq. 5 gives the increase in the culture's dry-weight concentration, $C$, which is determined for each time step, $\Delta t$, of the Lagrangian trajectory calculation by

$$
C(t+\Delta t)=C(t) \exp (\mu(t) \Delta t)
$$

where $\mu(t)$ is determined using the previous biological modeling.

Coupling between the trajectory calculation and the biological model is shown in Figure 13, where a single light-chamber passage is presented for the swirl motion and for the Grolux fluorescent tube. In this example, the dry-weight concentration of the culture at the entrance of the light chamber is set to a value at which a dark region occurs, namely, $C=1.8$ $\mathrm{g} \cdot \mathrm{L}^{-1}$. For each successive position of the microorganism trajectory (Figure 13a), the light received by the cell is determined using Eq. 4 (Figurc 13b) and the resulting growth rate deduced from the biological model described in the previous section (Figure 13c). This results in an increase in the dryweight concentration (Figure 13d). This is a step-by-step simulation wherein flow field, light availability, and growth inter-
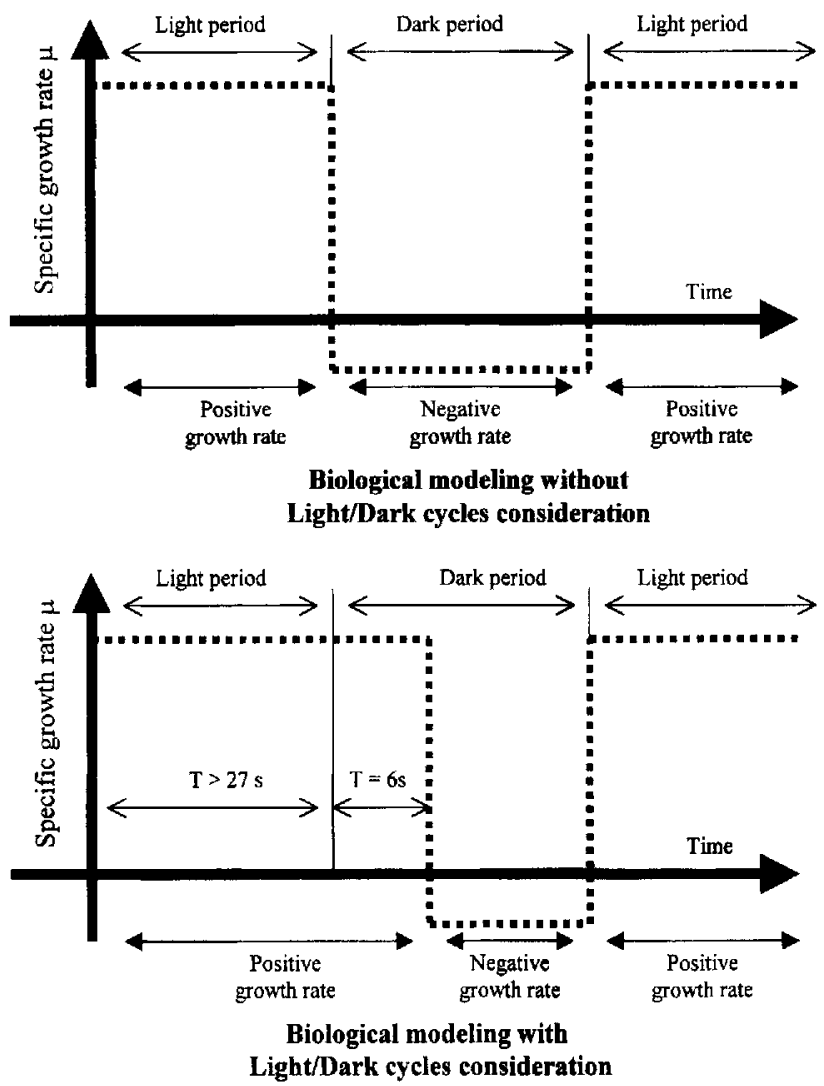

Figure 12. Light/dark cycles consideration in the biological modeling. 


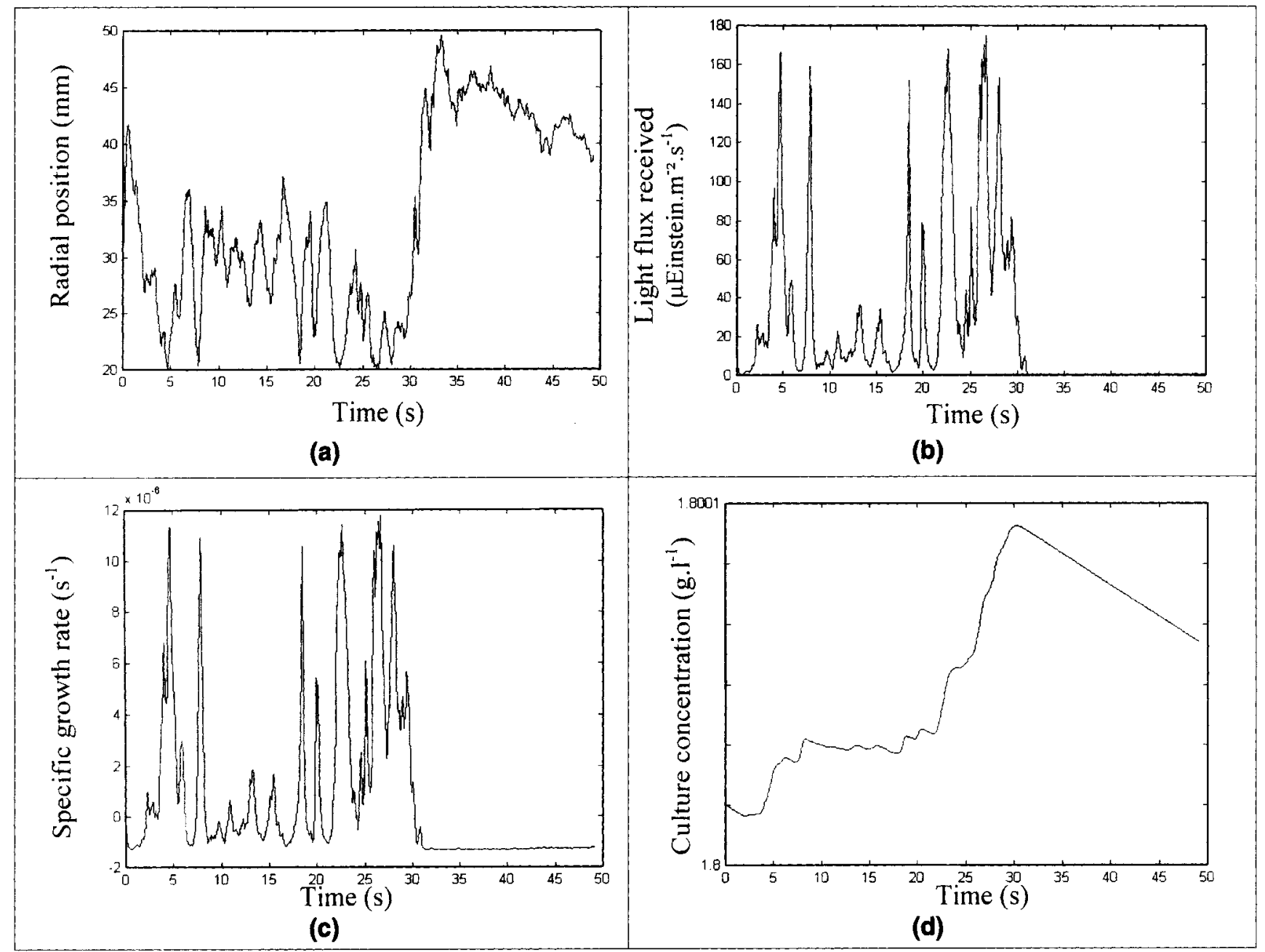

Figure 13. Example of progressive determination of the culture growth using the Lagrangian approach.

(a) Radial displacement of the microalga obtained with Lagrangian trajectory determination; (b) light received by the microalga calculated using Eq. 4 corresponding to the radial displacement; (c) photosynthetic response of the microalga obtained using the biological model; (d) evolution of the culture dry-weight concentration deduced from Eq. 6 and the biological model.

act: for each position of the microalga, the resulting increase in dry-weight concentration (Eq. 6) changes the light distribution in the culture (Eq. 4), which modifies the biological growth response $\mu(I)$, and thus the concentration evolution (Eq. 6). For this example, it can be stated that the microalga stays deep in the culture longer than $30 \mathrm{~s}$, receiving too little photonic energy to sustain a sufficient photosynthetic growth activity, which leads to negative values of the growth rate, and thus a decrease in culture concentration.

Complete batch culture simulations in the photobioreactor are achieved by adding loop circulation to the process and repeating the trajectory calculations, as described by the global algorithm given in Figure 14. The evolution of the culture's dry-weight concentration is simulated by considering the successive passages in the eight light chambers, alternatively set with both types of light sources, followed next by the culture's entry into the front side of the process, where no light is supplied. This last part of the process is thus considered to be a dark region, which has a residence time of $T_{d}=Q / V_{d}$, where $Q$ is the flow rate in the process and $V_{d}=22 \mathrm{~L}$ is the front-side volume. Simulation being achieved for $Q=1,190$
$\mathrm{L} \cdot \mathrm{h}^{-1}, T_{d}$ is thus a fixed period of $66 \mathrm{~s}$. For each new light chamber crossed, the radial and circumferential positions in the entrance of the annular geometry are randomly initialized before applying the Lagrangian calculation method of a microalga trajectory.

Batch-type cultures were simulated for both axial and swirl motions by determining the increase in dry-weight concentration until a limited value of concentration was reached. The time step, $\Delta t$, needed for the Lagrangian calculation of successive microalga position was set at $\Delta t=0.01 \mathrm{~s}$, which was found to give accurate results (Pruvost et al., 2002), and the value of the culture's dry-weight concentration was initialized to $C_{0}=0.1 \mathrm{~g} \cdot \mathrm{L}^{-1}$.

\section{Results and Discussion}

Microalgae culture growth has been simulated (Figure 15) by considering both axial and swirling flows in the light chambers. Each curve is obtained by simulating around 100,000 passages in the light chambers. To demonstrate the influence of light/dark cycles in the biological model, an additional 
simulation was made using the swirling motion, but without taking the light/dark cycles into account.

A typical batch culture evolution is represented on the different simulation curves: an initial exponential growth, followed by the effects of limited available light, which decreases the growth rate until the culture's dry-weight concentration is finally stabilized. The amount of available light decreases with the increase in the culture's dry-weight concentration, until the attenuation is too high to give sufficient light to microalgae to ensure their photosynthetic growth.

Light/dark cycles have a negligible effect on microalgae growth (Figure 15). Only a slight difference can be observed between both curves. Due to the ability of microalgae to remain in dark zones without weight loss, considering the effects of the light/dark cycles leads naturally to a better global culture growth rate, but only in a poor manner and for a restricted part of the growth curve: no influence is seen for low and high culture concentrations. For a small concentration of organisms at the beginning of the culture's growth, the light is not yet a limiting patameter, while the amount of light received by microalgae is greatly reduced for high concentrations. But the biological modeling assumes that an earlier period of illumination greater than $27 \mathrm{~s}$ is necessary to ensure no negative growth rate for the culture. This condition is not

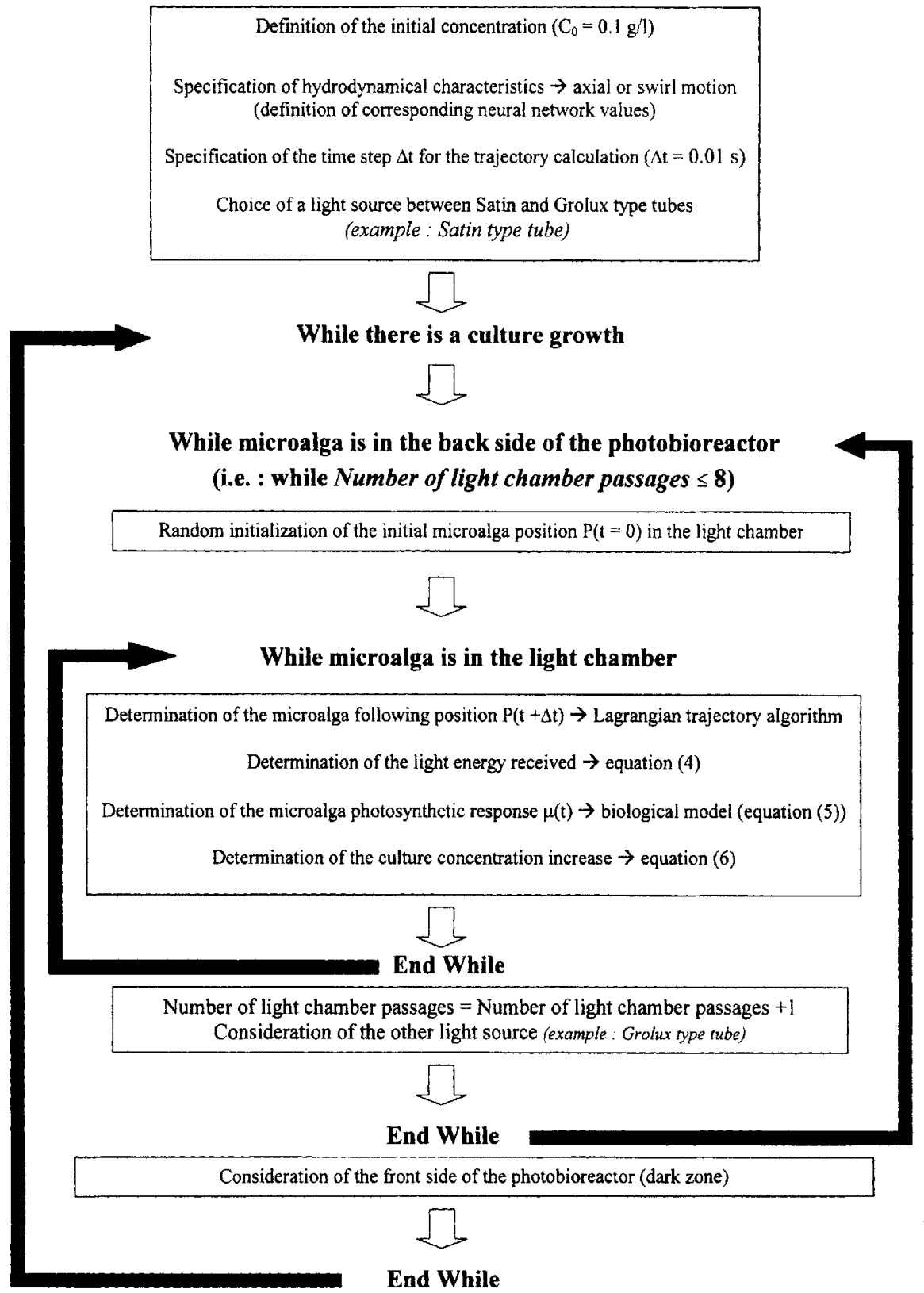

Figure 14. Global algorithm for batch culture simulations. 


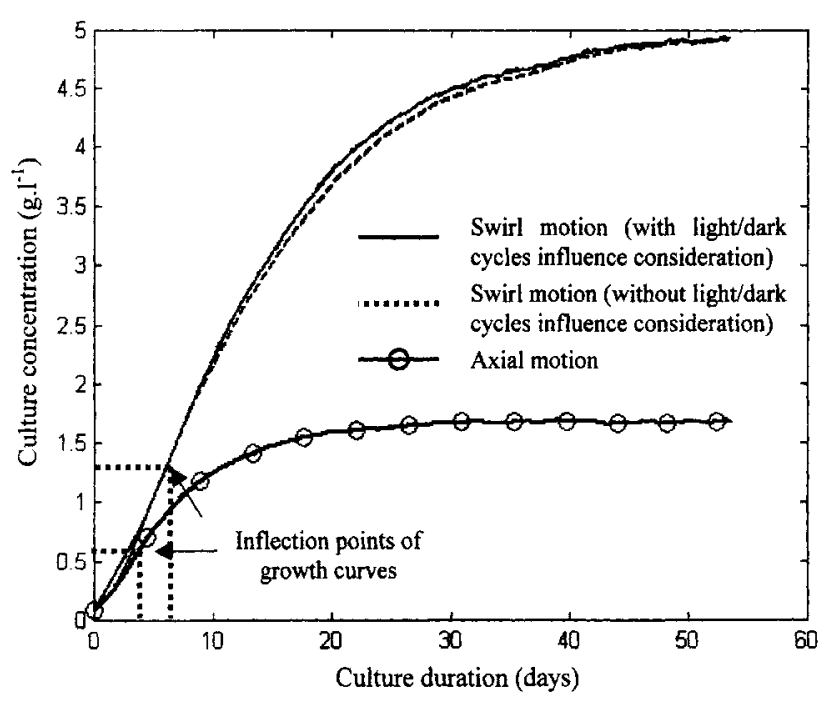

Figure 15. Batch cultures simulations with both axial and swirling motion in light chambers.

repeated in large culture concentrations, so considerations of light/dark cycles have no effect on the culture growth. Obviously, modeling is dependent on time values chosen to represent the influence of the D1 protein. But comparison of the curves obtained with and without light/dark cycles shows that this aspect has very little influence. On the contrary, results achieved with both hydrodynamical conditions are very different. The flow effect on microalgae growth is mainly linked to the trajectories involved, which provide different conditions for cell renewal in the presence of the light supply.

By considering the results shown in Figure 15 for both hydrodynamical conditions, the advantage of the swirling flow over the axial one is clearly observed. This demonstrates that hydrodynamical conditions have a fundamental influence on the culture growth. Three-dimensional flows, like the swirling one, cause trajectories that allow the culture to grow faster, while enhancing the value of the limiting dry-weight concentration. As shown in Figure 4 for axial flow, a microalga entering the light chamber in an initial position located deep in the culture remains at the same distance from the light source during its journey. Thus, if the culture is concentrated, the weight loss is important. This is not the case in swirling flow as the three-dimensional trajectory allows each microalga to receive light even in a concentrated culture. This explains why the difference between both flows increases with the dryweight concentration: the light attenuation effects are greater for high concentrations. The axial flow does not allow the process to be used optimally, and one way to improve the performance is to impose hydrodynamical conditions that move in the direction of the light gradient. Cell renewal in front of the light source allows sufficient light to ensure good photosynthesis conditions, even when the light doesn't penetrate very deep into the culture. We can conclude that a 3-D flow delays the appearance of the light limitation, which is observed at the inflection points in Figure 15, characterizing the change in growth evolution. For the axial flow, light appears as a limiting parameter for dry-weight concentrations greater than $0.6 \mathrm{~g} \cdot \mathrm{L}^{-1}$, while a value of $1.3 \mathrm{~g} \cdot \mathrm{L}^{-1}$ is obtained for the swirling flow.

\section{Conclusion}

Simulations of batch cultures of $P$. purpureum were achieved in a photobioreactor with circular light chambers. Attention was focused on the effects of hydrodynamical conditions on the light availability and the growth of microalgae by comparing two kinds of flow: an axial one, which induces a poor mixing although it is commonly used in such geometries, and a swirling decaying flow, which generated a 3-D motion. A Lagrangian approach was used to follow microalga displacement in the light chamber to determine the amount of light received by the microorganisms. The Beer-Lambert law, modified to consider light diffusion and the scattering effects of the microalgae culture, was used to calculate light distribution as a function of the culture's dry-weight concentration. Finally, a biological model that included light/dark cycles adapted for the $P$. purpureum microalgae was proposed and then related to the trajectory and light distribution calculations.

Comparison between axial and swirling motions has shown the advantage of generating 3-D flow fields to decrease the effects of the limited light when the culture concentration increases. By investigating the swirl motion with and without including light/dark cycles, it appeared that this particular photosynthetic behavior of the microalgae doesn't have much of an effect on the resulting growth. By allowing cell movement along the light gradient, the light absorbed by each microalga is improved in the swirl flow, which lessens the effects of light attenuation and leads to a higher limiting value for the dry-weight concentration.

The photobioreactor model focused on the effect of the change in flow conditions on available light for the culture. Under actual conditions, the culture is exposed to other limiting parameters, such as nutrients. But, because the influence of each limiting parameter remains difficult to study in nature, simulations are essential for understanding the operation of photobioreactors. For example, increasing the growth rate using 3-D flows alters the cells' nutritional needs, which then could replace light as the limiting parameter. The microalgae culture is thus the result of an overall set of interacting parameters, all of which are not completely identified. Simulation is thus complementary to experimental studies on microalgae culture and provides a better understanding of a selected parameter, which is essential for fully understanding the overall complex running of a photobioreactor and allows optimization of such a process.

\section{Literature Cited}

Aro, E. M., I. Virgin, and B. Andersson, "Photoinhibition of Photosystem II. Inactivation, Protein Damage and Turnover," Biochim. Biophys. Acta, 1143, 113 (1993).

Baldyga, J., and R. Pohorecki, "Influence of Turbulent Mechanical Stresses on Microorganisms," ASME Appl. Mech. Rev., 51(1), 121 (1998).

Bradshaw, P., An Introduction to Turbulence and Its Measurement, Pergamon Press, Oxford (1971).

Carlozzi, P., and G. Torzillo, "Productivity of Spirulina in a Strongly Curved Outdoor Tubular Photobioreactor," Appl. Microbiol. Biotechnol., 45(1-2), 18 (1996).

Cassano, A., C. Martin, R. Brandi, and O. Alfano, "Photoreactor Analysis and Design: Fundamentals and Applications," Ind. Eng. Chem. Res., 34, 2155 (1995).

Chen, K. W., and Y. K. Lee, "Growth of Chlorella Outdoors in a Changing Light Environment,”J. Appl. Phycol., 9, 425 (1997). 
Cornet, J. F., C. G. Dussap, and J. B. Gros, "A Simplified Monodimensional Approach for Modeling Coupling Between Radiant Light Transfer and Growth Kinetics in Photobioreactors," Chem. Eng. Sci., 50(9), 1489 (1995).

Cornet, J. F., C. G. Dussap, and J. B. Gros, "Kinetics and Energetics of Photosynthetic Micro-Organisms in Photobioreactors-Applicarion to Spindina Growth," Adv. Biochem. Eng. Biotechnol, , 59, 153 (1998).

Dermoun, D., D. Chaumont, J. M. Thebault, and A. Dauta, "Modeling of Growth of Porphyridium cruentum in Connection with Two Interdependent Factors: Light and Temperature," Bioresour. Technol., 42, 113 (1992).

Fernandez, A. F. G., G. F. Camacho, S. J. A. Perez, F. J. M. Sevilla, and M. E. Grima, "A Model for Light Distribution and Average Solar Irradiance Inside Outdoor Tubular Photobioreactors for the Microalgal Mass Culture," Biotechnol. Bioeng., 55(5), 701 (1997).

Frolich, B. T., I. A. Webster, M. M. Ataai, and M. L. Shuler, "Photobioreactors: Models for Interaction of Light Intensity, Reactor Design, and Algal Physiology," Biotechnol. Bioeng. Symp., 13, 331 (1983).

Grima, M. E., A. F. G. Fernandez, G. F. Camacho, and Y. Chisti, "Photobioreactors: Light Regime, Mass Transfer, and Scaleup," J. Biotechnol., 70, 231 (1999).

Grima, M. E., F. J. M. Sevilla, J. A. S. Perez, and F. G. Camacho, "A Study on Simultaneous Photolimitation and Photoinhibition in Dense Microalgal Cultures Taking into Account Incident and Averaged Irradiances," J. Biotechnol., 45, 59 (1996).

Grobbelaar, J. U., "Turbulence in Mass Algal Cultures and the Role of Light/Dark Fluctuations," J. Appl. Phycol., 6, 331 (1994).

Grobbelaar, J. U., L. Nebdal, and V. Tichy, "Influence of High Light/Dark Fluctuations on Photosynthetic Characteristics of Microalgae Photoacclimated to Different Light Intensities and Implications for Mass Algal Cultivation," J. Appl. Phycol., 8, 335 (1996),

Gudin, C., and D. Chaumont, "Cell Fragility: The Key Problem of Microalgae Mass Production in Closed Photobioreactors," Biores. Technol., 38(2-3), 145 (1991).

Gupta, A. K., D. C. Lilley, and N. Syred, Swirl Flows, Abacus Press, Cambridge (1984)

Janssen, M. G. J., T. C. Kuijpers, B. Veldhoen, M. B. Ternbach, J. Tramper, L. R. Mur, and R. H. Wijffels, "Specific Growth Rate of Chlamydomonas reinhardtii and Chlorella sorokiniana Under Medium Duration Light/Dark Cycles 13-87 s," J. Biotechnol., 70, 323 (1999).

Jaouen, P., L. Vandanjon, and F. Quéméneur, "The Shear Stress of Microalgal Cell Suspensions (Tetraselmis suecica) in Tangential Flow Filteration Systems: The Role of Pumps," Biores. Technol, 68, 149 (1999).

Lee, C. G., "Calculation of Light Penetration Depth in Photobioreactors," Biotechnol. Bioprocess Eng., 4, 78 (1999).

Lee, K., and C. G. Lee, "Effect of Light/Dark Cycles on Wastewater Treatments by Microalgae," Biotechnol. Bioprocess Eng., 6, 194 (2001).

Legentilhomme, P., H. Aouabed, and J. Legrand, "Developing Mass Transfer in Swirling Decaying Flow Induced by Means of a Tangential Inlet," Chem. Eng. J., 52, 137 (1993).

Legentilhomme, P., and J. Legrand, "Overall Mass Transfer in Swirling Decay Flow in Annular Electrochemical Cells," J. Appl. Electrochem., 20, 216 (1990).

Legentilhomme, P., and J. Legrand, "The Effects of Iniet Conditions on Mass Transfer in Annular Swirling Decaying Flow," Int. J. Heat Mass Transfer, 34, 1281 (1991).

Merchuk, J. C., M. Ronen, S. Giris, and S. Arad, "Light/Dark Cycles in the Growth of the Red Microalga Porphyridium sp.," Biotechnol. Bioeng., 59(6), 705 (1998).
Miller, R. L., A. G. Fredrickson, A. H. Brown, and H. M. Tsuchiya, "Hydromechanical Method to Increase Efficiency of Algal Photosynthesis," Ind. Eng. Chem. Proc. Des. Dev., 3, 134 (1964).

Mitsuhachi, S., K. Hosaka, E. Tomonaga, H. Muramatsu, and K. Tanishita, "Effects of Shear Flow on Photosynthesis in a Dilute Suspension of Microalgae," Appl. Microbiol. Biotechnol., 42, 744 (1995).

Muller-Feuga, A., "Growth as a Function of Rationing: A Model Applicable to Fish and Microalgae,"J. Exp. Mar. Biol. Ecol., 236, 1 (1998).

Muller-Feuga, A., R. Le Guédes, A. Hervé, and P. Durand, "Conparison of Artificial Light Photobioreactors and Other Production Systems Using Porphyridium cruentum," J. Appl. Phycol, 10, 83 (1998a).

Muller-Feuga, A., R. Baron, R. Le Guédes, and E. Michel, "Theoretical Assessment of Productivity in Photobioreactors by Radiative Transfer Approach," Marine Micro-Organisms for Industry, Ifremer Editions, Brest, France, p. 194 (1998b).

Muller-Feuga, A., J. Legrand, J. Pruvost, P. Legentilhomme, and R. Le Guédes, "Procédé pour Améliorer le Transfert dans une Chambre de Bioréaction," Ifremer and Nantes-University French Patent No. 00-08587 (2000).

Ormancey, A., and J. Martinon, "Prediction of Particle Dispersion in Turbulent Flows," Phys. Chem. Hydrodynam., 5(3/4), 229 (1984).

Powell, M. J. D., "Radial Basis Functions for Multivariate Interpolation: A Review," Algorithms for Approximation, J. C. Mason and M. G. Cox, eds., Clarendon Press, Oxford (1987).

Pruvost, J., J. Legrand, and P. Legentilhomme, "Transfert Photonique dans un Photoréacteur à Écoulement Tourbillonnaire," Can. J. Chem. Eng., 77, 869 (1999).

Pruvost, J., J. Legrand, P. Legentilhomme, and L. Doubliez, "Particle Image Velocimetry Investigation of the Flow-Field of a 3D Turbulent Annular Swirling Decaying Flow Induced by Means of a Tangential Inlet," Exp. Fluids, 29(3), 291 (2000).

Pruvost, J., J. Legrand, P. Legentilhomme, and A. Muller-Feuga, "Lagrangian Trajectory Model for Turbulent Swirling Flow in an Annular Cell. Comparison with Residence Time Distribution Measurements," Chem. Eng. Sci., 57(7), 1205 (2002).

Pruvost, J., J. Legrand, and P. Legentilhomme, "Three-Dimensional Swirl Flow Velocity-Field Reconstruction Using a Neural Network with Radial Basis Functions," J. Fluids Eng., 123, 920 (2001).

Pulz, O., and K. Scheibenbogen, "Photobioreactors: Design and Performance with Respect to Light Energy Input," Advances in Biochemical Engineering Biotechnology, 59, 123 (1998).

Richmond, A., Handbook of Microalgal Mass Culture, CRC Press, Boca Raton, FL (1986).

Richmond, A., "Efficient Utilization of High Irradiance for Production of Photoautotrophic Cell Mass: A Survey,"J. Appl. Phycol., 8, 381 (1996).

Thomas, W. H., and C. H. Gibson, "Effects of Small Turbulence on Microalgae," J. Appl. Phycol, 2, 71 (1990).

Vandanjon, L., N. Rossignol, P. Jaouen, J. M. Robert, and F. Quéméneur, "Effects of Shear on Two Microalgae Species. Contribution of Pumps and Valves in Tangential Flow Filtration Systems," Biotechnol. Bioeng., 63(1), 1 (1999).

Vonshak, A., Spirulina platensis (Artrospira): Physiology, Cell-Biology and Biotechnology, Taylor \& Francis, London (1997).

Yokota, T., M. Hizume, T. Ohtake, and K. Takahashi, "A New Growth Kinetic Model for Photoautotrophic Microalgae Culture," J. Chem. Eng. Jpn., 27(3), 399 (1994). 